\title{
Терагерцовое стимулированное излучение при оптическом резонансном возбуждении германия, легированного мелкими донорами
}

\author{
(C) Р.Х. Жукавин \\ Институт фризики микроструктур Российской академии наук, \\ 607680 Нижний Новгород, Россия \\ E-mail: zhur@ipmras.ru \\ Поступила в Редакцию 12 апреля 2021 г. \\ В окончательной редакции 19 апреля 2021 г. \\ Принята к публикации 19 апреля 2021 г.
}

Рассмотрены механизмы, ответственные за терагерцовое стимулированное излучение при резонансном внутрицентровом возбуждении мелких доноров в германии: инверсионный лазерный механизм и электронное вынужденное комбинационное рассеяние света. Проведены оценки сечения электронного вынужденного комбинационного рассеяния света в случае резонансного возбуждения нечетных уровней мелкого донора мышьяка в объемном германии. Проведены расчеты выходной интенсивности при резонансном возбуждении германия, легированного мышьяком. Показано, что при интенсивности, превышающей пороговую для электронного вынужденного комбинационного рассеяния света, должна наблюдаться конкуренция механизмов, приводящая к снижению интенсивности инверсионного лазерного механизма, что может быть обнаружено по зависимости выходной интенсивности от времени.

Ключевые слова: германий, доноры, инверсия, терагерцовое стимулированное излучение, вынужденное комбинационное рассеяние.

DOI: 10.21883/FTP.2021.09.51285.12

\section{1. Введение}

В настоящее время примесные центры в таких полупроводниках как германий и кремний вновь оказались в фокусе исследований. Это обстоятельство связано с возможностью создания различных устройств, использующих свойства, связанные с возможностью манипуляции когерентными состояниями методами квантовой оптики [1] и формированием инвертированных распределений в таких системах $[2,3]$. В этом же ключе можно рассматривать возрожденный интерес к экситонам в полупроводниках $[4,5]$. Этому способствует прогресс, достигнутый в степени очистки полупроводников от посторонних примесей и изотопов, а также развитие экспериментальной базы. В частности, использование лазеров на свободных электронах позволило определить время продольной релаксации $T_{1}$ для состояний доноров и акцепторов в кремнии и германии [6-8], время поперечной релаксации $T_{2}$ доноров кремнии [1], а также провести эксперименты по наблюдению фотонного эха при резонансном возбуждении доноров в кремнии. Полученные данные свидетельствуют о том, что время $T_{1}$ для состояния $2 p_{0}$ мышьяка в германии в несколько раз превышает аналогичные параметры для доноров в кремнии. С одной стороны, экспериментальные данные находятся в качественном согласии с результатами работы [3], где были оценены времена релаксации возбужденных состояний доноров мышьяка в германии, и показана возможность получения инверсии населенности на переходах $2 p-1 s\left(T_{2}\right)$. Это обстоятельство позволило на- деяться на возможность получения некоторых эффектов, наблюдаемых ранее в кремнии, в частности генерации стимулированного терагерцового излучения при оптическом возбуждении. С другой стороны, полученные экспериментальные данные [8] указывают, что времена возвращения электронов при возбуждении состояний, лежащих выше уровня $2 p_{0}$, оказываются короткими, что указывает на существование канала прямой релаксации на переходах в основное состояние при приближении к энергии TA фононов германия ( 10 мэВ). На данном этапе существует проблема адекватного описания волновой функции основного состояния мышьяка в германии, так как мышьяк обладает энергией ионизации, превышающей на 40\% энергию, рассчитанную в приближении эффективной массы, что говорит о значительном долинно-орбитальном взаимодействии. Таким образом, наличие подобного канала релаксации может приводить к снижению ожидаемого коэффициента усиления в случае использования фотоионизирующей накачки. В этой связи с целью проверки возможности получения лазерного эффекта актуальным является использование перестраиваемого по частоте источника накачки с возможностью резонансного возбуждения того или иного уровня донора. При этом, как известно, внутрицентровое возбуждение может приводить к развитию не только лазерного механизма инверсионного типа, но и электронного вынужденного комбинационного рассеяния (ЭВКР). В частности, существование обоих эффектов было зарегистрировано в кремнии, легированном мелкими донорами, и продемонстрирована 
возможность конкуренции между этими механизмами при определенных условиях наблюдения [9]. Данная работа представляет результат рассмотрения совместного действия инверсионного и безынверсного механизмов при внутрицентровом возбуждении доноров мышьяка в кремнии.

\section{2. Модель и результаты}

Вычисление коэффициента усиления при оптическом возбуждении дискретной системы уровней является хорошо известной задачей, которая может решаться, в частности, как в рамках системы балансных уравнений, так и матрицы плотности, причем последний метод особенно актуален при когерентной накачке. Далее будут приведены результаты анализа на основе решения балансных уравнений, что позволит сделать простые выводы, хотя и достаточно качественного порядка.

Расчет населенностей и интенсивности излучения проводился в рамках решения балансных уравнений, ограничившись трехуровневой схемой по аналогии с работой [9]. При расчете применялось решение системы балансных уравнений для трех уровней 1,2 и 3 (основное состояние донора $1 s\left(A_{1}\right), 1 s\left(T_{2}\right), 2 p_{0} / 2 p_{ \pm}$ соответственно, рис. 1) с населенностями $n 1, n 2, n 3$, а также фотонов, имеющих энергии, близкие к энергии перехода 3-2. В качестве параметров использовались интенсивность возбуждения, добротность резонатора (соответствующее время жизни фотона в резонаторе $\left.10^{-8} \mathrm{c}\right)$, времена релаксации уровней 3 и 2, взятые из эксперимента [8]: 600 и 100 пс соответственно и, как уже было сказано выше, близкие к временам, полученным в результате расчета вероятностей испускания акустических фононов. Равновесная населенность возбужденных состояний полагалась равной нулю, т.е. результаты расчета можно считать применимыми в диапазоне температур, при которых $k T$ много меньше энергии связи примеси (14.18 мэВ).

Сечения для перехода 3-2 и 1-3 были взяты с использованием данных статьи [10]. Сечение ЭВКР рассчитывалось в приближении резонансного случая в соответствии с подходом работы [11] с использованием матричных элементов переходов 3-2 и $1-3$ работы [10]. Получены зависимости сечений ЭВКР усиления при резонансной накачке уровней $2 p_{0}$ и $2 p_{ \pm}$от плотности потока возбуждения (рис. 2). При одинаковой интенсивности возбуждения сечение ЭВКР для случая резонанса с $2 p_{ \pm}$значительно (на 2 порядка) превышает таковое для $2 p_{0}$. Эта разница в основном обусловлена отличием в матричных элементах участвующих переходов (фактор 3), входящих в степени 4 (пренебрегая отличием для состояний $1 s\left(A_{1}\right)$ и $1 s\left(T_{2}\right)$. Величина сечения усиления ЭВКР достигает $10^{-14} \mathrm{~cm}^{2}$ при интенсивностях $\sim 4$ кВт/см ${ }^{2}$, что сравнимо с сечением поглощения на участвующих переходах. Сечение рассеяния пропорционально интенсивности накачки, что позволяет увеличить

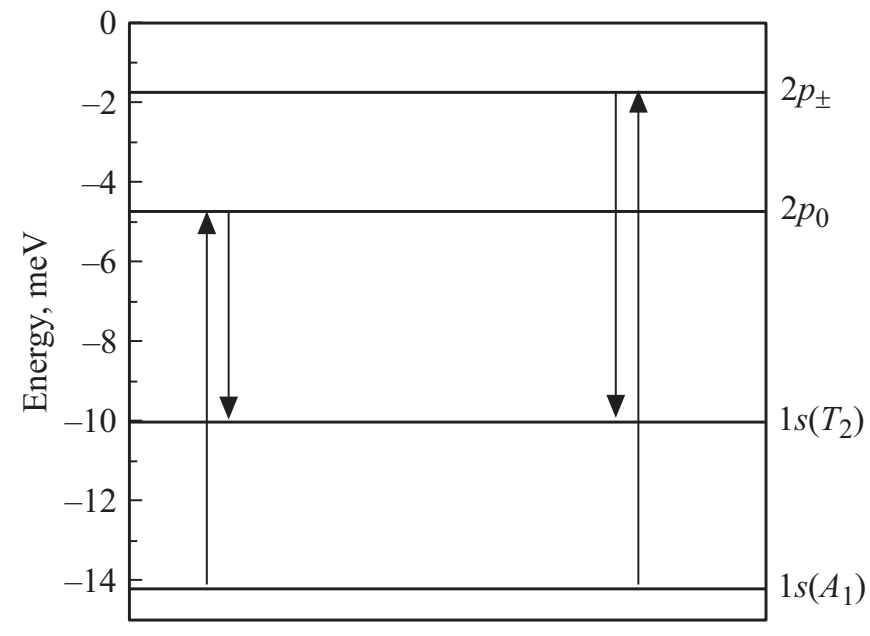

Рис. 1. Уровни мышьяка в германии, принятые к рассмотрению в случае резонансной накачки. Стрелки, направленные вверх, соответствуют квантам возбуждения, вниз - квантам излучения.

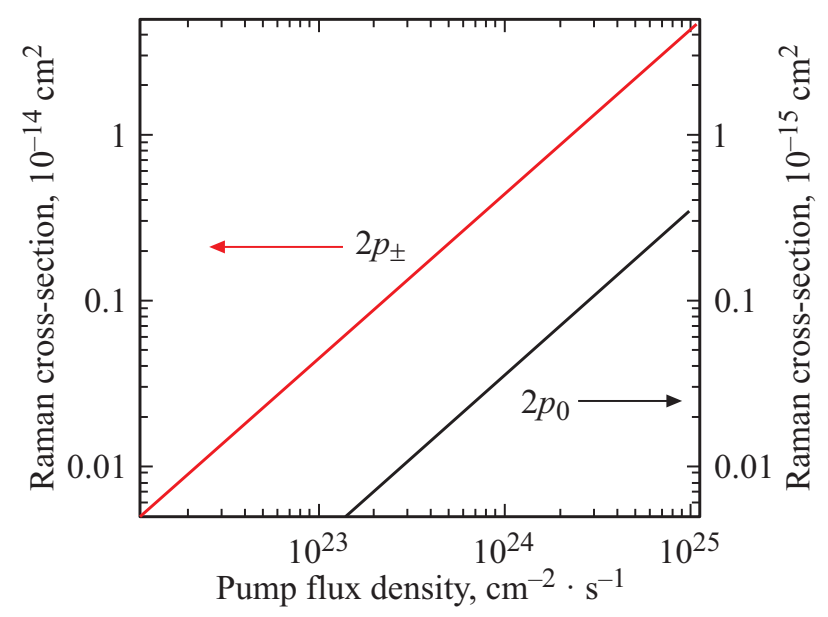

Рис. 2. Зависимость сечения ЭВКР от плотности потока возбуждения для двух частот возбуждения для ширины линии переходов, равной 0.1 мэВ.

коэффициент усиления, однако в резонансном случае это приведет также и к сопутствующей ионизации примеси, поэтому обычно рассматривают случай отстройки от резонанса [11]. Для оценки величины сечения рассеяния вне резонанса удобно отойти от трехуровневого приближения и использовать величину, рассчитанную во втором порядке теории возмущений с учетом реального положения нескольких уровней примеси. Рис. 3 представляет результат расчета сечения рассеяния в зависимости от частоты падающего излучения при фиксированной интенсивности возбуждения. В рамановской сумме учтены матричные элементы [10] для переходов между состояниями $1 s$ и несколькими нечетными состояниями, имеющими наибольшие значения $\left(2 p_{0}, 3 p_{0}\right.$, $\left.4 p_{0}, 2 p_{ \pm}, 3 p_{ \pm}, 4 p_{ \pm}\right)$. Это естественным образом привело 


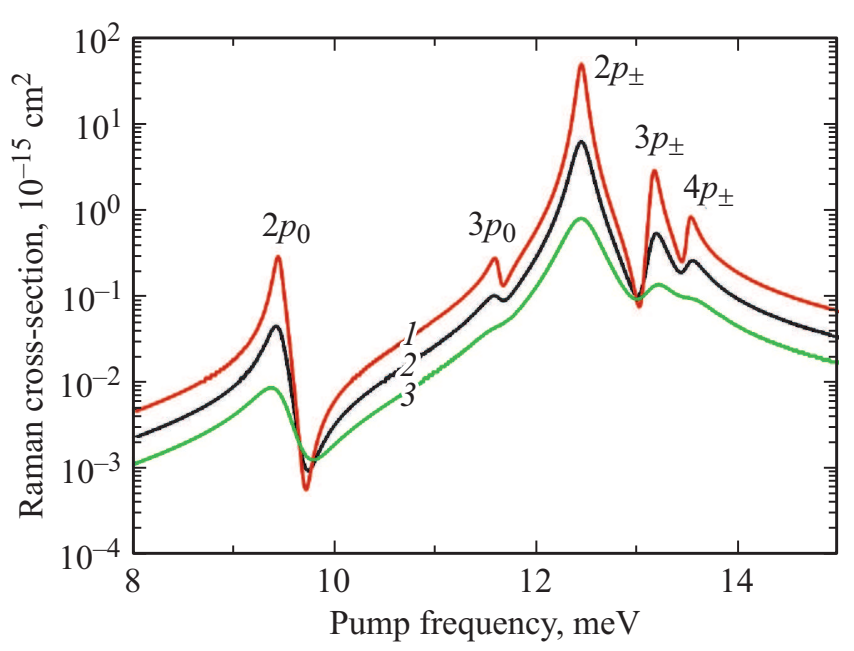

Рис. 3. Зависимость сечения ЭВКР от частоты возбуждения при нескольких значениях уширения линий, мэВ: 0.05 (1), 0.1 (2), 0.2 (3). Интенсивность возбуждения 4 кВт/см². (Цветной вариант рисунка представлен в электронной версии статьи).

к возникновению нескольких пиков, соответствующих резонансам, ширина и амплитуда которых зависят от ширины линии поглощения и соответствующих матричных элементов. Из рис. 3 видно, что накачка уровня $2 p_{0}$ (квант накачки $\sim 9.5$ мэВ) не дает максимальное сечение ЭВКР, однако стоит отметить, что с точки зрения проведения экспериментальной проверки важным фактором является то, что энергия связи этого уровня среди нечетных является самой большой, что минимизирует процессы фототермоионизации при резонансной накачке. Напротив, резонансное возбуждение уровня $2 p_{ \pm}$ $(\sim 12.5$ мэВ) позволяет получить максимальное сечение усиления, однако фактор термоионизации может оказаться критическим. Естественно, что отстройка от резонанса устраняет данный фактор, хотя и сопровождается снижением сечения. Таким образом, достаточно высокие значения для сечений усиления ЭВКР вызваны применением упрощенного подхода и могут быть рассмотрены в качестве верхней оценки эффективности механизма ЭВКР. Более корректный анализ может состоять в рассмотрении коэффициента усиления с учетом ионизации накачиваемых состояний, как в рамках оптических переходов, так и термической ионизации, что очевидным образом снизит эффективность процесса рассеяния.

Так как при резонансной накачке возможно развитие излучения по инверсионному и безынверсному механизмам, возможно существование конкуренции этих двух механизмов, а значит, сравнение малосигнальных коэффициентов усиления не информативно, в случае если усиление ЭВКР (произведение сечения на населенность основного состояния) превышает потери, хотя и уступает усилению ИЛМ. В дальнейших расчетах предполагалось, что излучение накачки обладает импульсной структурой. В качестве примера для рассмотрения был выбран стандартный режим лазера на свободных электронах FELIX (см., например, [9]), где импульсы излучения длительностью 10 пс следуют с периодом 1 нс. Рис. 4 представляет результат расчета интенсивности выходного излучения в результате действия ИЛМ и ЭВКР механизмов без учета пространственного распределения. Приведены два случая, отличающиеся интенсивностью возбуждения $\left(4 \mathrm{\kappa BT} / \mathrm{cm}^{2}\right)$ при накачке уровня $2 p_{ \pm}$. Энергия кванта излучения соответствует энергии перехода $2 p_{ \pm}-1 s\left(T_{2}\right)$. В первом случае (рис. $\left.4, a\right)$ интенсивность выбрана так, чтобы обеспечить равенство сечений усиления в рамках обоих механизмов усиления. При выбранных параметрах развивается излучение ИЛМ. Увеличение интенсивности в 2 раза (рис. $4, b$ ) приводит
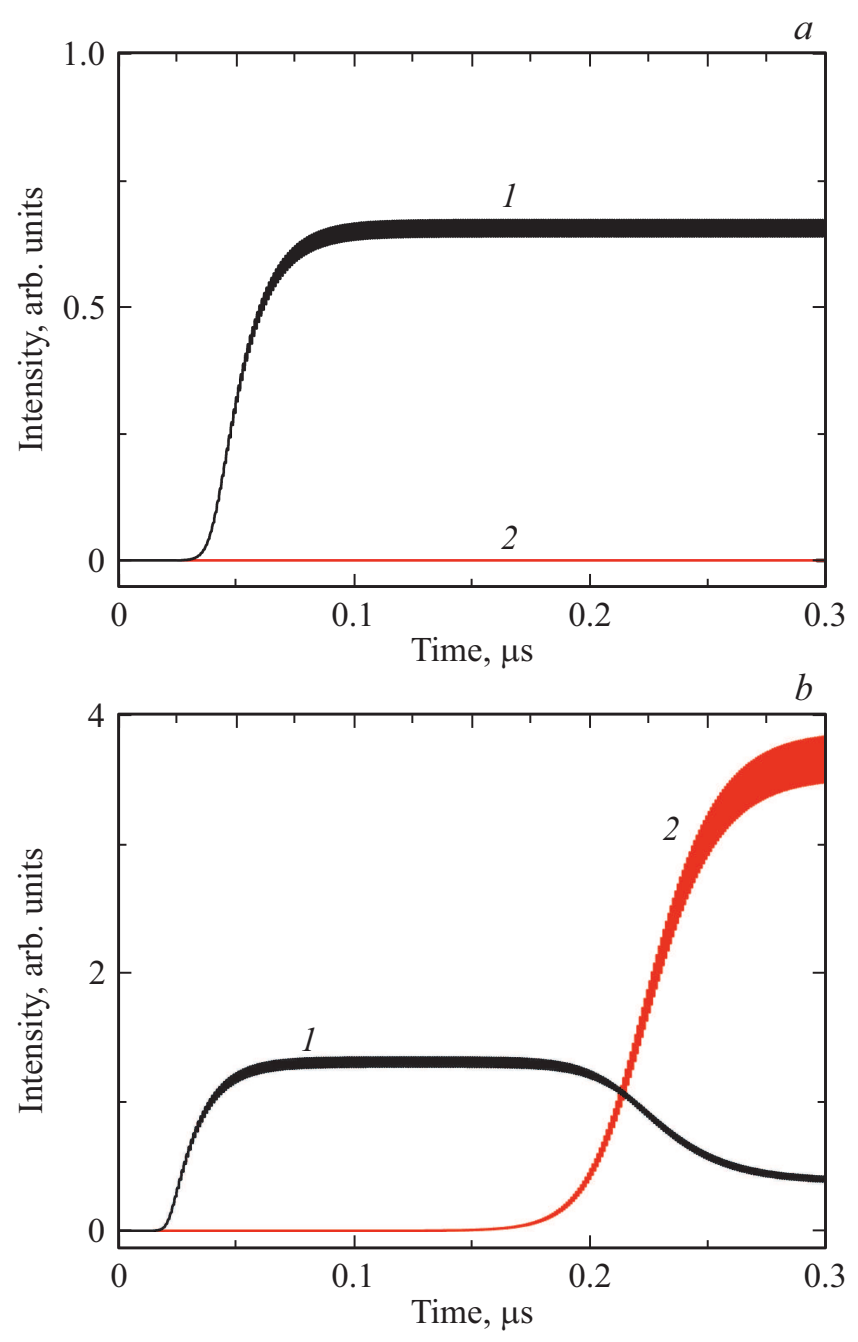

Рис. 4. Интенсивность излучения для инверсионного (1) и безынверсного (2) типов генерации для двух интенсивностей накачки при решении 3 -уровневой системы для уровня $2 p_{ \pm}$: $4(a)$ и 8 кВт/см ${ }^{2}(b)$. Энергия кванта излучения соответствует энергии перехода $2 p_{ \pm}-1 s\left(T_{2}\right)$. Временна́я структура излучения накачки представляла собой макроимпульс, состоящий из микроимульсов длительность 10 пс и частотой повторения 1 ГГц. „Включение“ возбуждения происходит в момент времени $t=0$. 
к удвоению сечения ЭВКР и генерации в рамках двух механизмов, причем излучение ЭВКР возникает с заметной задержкой относительно инверсионного лазера и приводит к снижению интенсивности излучения последнего. Данный результат можно легко понять, если учесть импульсный характер накачки. Необходимым условием развития генерации является превышение усиления за время его существования над потерями за весь период. Если принять за эффективные потери величину $0.01 \mathrm{~cm}^{-1}$, то при концентрации $10^{14} \mathrm{~cm}^{-3}$,граница“ существования ЭВКР оказывается в районе 4 кВт/см ${ }^{2}$ в случае возбуждения состояния $2 p_{ \pm}$и $0.4 \mathrm{MBT} / \mathrm{cm}^{2}$ для $2 p_{0}$. При меньших мощностях возбуждения реализуется только инверсионный лазерный механизм. Качественно, можно считать, что время усиления в рамках инверсионного механизма определяется временем жизни верхнего лазерного уровня $(800 \pi \mathrm{n})$, в то время как усиление ЭВКР существует лишь во время микроимпульса накачки (в данном случае 10 пс). При превышении порога развивается также и генерация по механизму ЭВКР, при этом конкуренция с инверсионным механизмом вызвана тем, что в акте ЭВКР не происходит заселение верхнего лазерного уровня. Последнее и приводит к снижению эффективности инверсионного механизма. Таким образом, наблюдение динамики развития генерации позволит определить существование эффекта конкуренции в активной среде.

\section{3. Заключение}

В данной работе был рассмотрен случай лазерной генерации при внутрицентровом возбуждении доноров мышьяка в германии с учетом вклада электронного вынужденного комбинационного рассеяния (рамановской генерации). Необходимость проведения расчетов диктуется наличием экспериментальных данных, говорящих о существовании каналов относительно быстрой релаксации верхних возбужденных состояний для мышьяка в германии, что может исключить возможность усиления и стимулированного излучения при использовании фотоионизирующей накачки примесных центров. Сделаны оценки сечения рамановского усиления как в резонансном случае, так и вне резонансов с уровнями донора. Были проведены модельные расчеты, которые показали, что при определенных и достижимых параметрах возможна лазерная генерация только в рамках инверсионного механизма, а также сосуществование инверсионного и безынверсного механизмов генерации в режиме конкуренции. Переход от одного режима к другому осуществляется увеличением интенсивности возбуждения.

\section{Финансирование работы}

Работа выполнена при поддержке РНФ (проект 19-72-20163).

\section{Конфликт интересов}

Автор заявляет, что у него нет конфликта интересов.

\section{Список литературы}

[1] K.L. Litvinenko, E.T. Bowyer, P.T. Greenland, N. Stavrias, J. Li, R. Gwilliam, B.J. Villis, G. Matmon, MLY. Pang, B. Redlich, A.F.G. van der Meer, C.R. Pidgeon, G. Aeppli, B.N. Murdin. Nature Commun., 6, 6549 (2015).

[2] S.G. Pavlov, R.Kh. Zhukavin, V.N. Shastin, H.-W. Hübers. Phys. Status Solidi B, 250, 9 (2013).

[3] В.В. Цыпленков, В.Н. Шастин. ФТП, 52, 1469 (2018).

[4] A.V. Andrianov, A.O. Zakhar'in. Phys. Status Solidi B, 256, 1800496 (2019).

[5] J. Heckötter, M. Freitag, D. Fröhlich, M. Aßmann, M. Bayer, M.A. Semina, M.M. Glazov. Phys. Rev. B, 96, 125142 (2017).

[6] N.Q. Vinh, B. Redlich, A.F.G. van der Meer, C.R. Pidgeon, P.T. Greenland, S.A. Lynch, G. Aeppli, B.N. Murdin. Phys. Rev. X, 3, 011019 (2013).

[7] N. Deßmann, S.G. Pavlov, A. Pohl, N.V. Abrosimov, S. Winnerl, M. Mittendorff, R.Kh. Zhukavin, V.V. Tsyplenkov, D.V. Shengurov, V.N. Shastin, H.-W. Hübers. Appl. Phys. Lett., 106, 171109 (2015).

[8] R.Kh. Zhukavin, K.A. Kovalevskii, Yu.Yu. Choporova, V.V. Tsyplenkov, V.V. Gerasimov, P.A. Bushuikin, B.A. Knyazev, N.V. Abroimov, S.G. Pavlov, H.-W. Hübers, V.N. Shastin. JETP Letters, 110, 677 (2019).

[9] S.G. Pavlov, N. Deßmann, B. Redlich, A.F.G. van der Meer, N.V. Abrosimov, H. Riemann, R.Kh. Zhukavin, V.N. Shastin, H.-W. Hübers. Phys. Rev. X, 8, 041003 (2018).

[10] P. Clauws, J. Broeckx, E. Rotsaert, J. Vennik. Phys. Rev. B, 38, 12377 (1988).

[11] J.B. Khurgin, G. Sun, L.R. Friedman, R.A. Soref. J. Appl. Phys., 78, 7398 (1995).

Редактор Г.А. Оганесян

\section{Terahertz stimulated emission under optical resonant excitation of germanium doped by shallow donors}

\section{R.Kh. Zhukavin}

Institute for Physics of Microstructures

Russian Academy of Sciences, 607680 Nizhny Novgorod, Russia

\begin{abstract}
The mechanisms responsible for terahertz stimulated radiation under resonant intracenter excitation of shallow donors in bulk germanium are considered to be the inversion laser mechanism (ILM) and electron stimulated Raman scattering (e-SRS). The e-SRS cross-section was estimated in the case of resonant excitation of odd levels of a shallow arsenic donor in germanium. The output intensity under resonant excitation of germanium doped with arsenic is calculated. It is shown that at an intensity exceeding the threshold for e-SRS, there should be a competition of mechanisms leading to a decrease in the intensity of ILM, which can be detected by the dependence of the output intensity on time.
\end{abstract}

